DOI: $10.17516 / 1997-1397-2021-14-1-87-97$

УДК $517.95+534.1+532.5 .031$

\title{
The Response of a Poroelastic Ice Plate to an External Pressure
}

\author{
Kristina N. Zavyalova* \\ Konstantin A. Shishmarev ${ }^{\dagger}$ \\ Altai State University \\ Barnaul, Russian Federation \\ Alexander A. Korobkin ${ }^{\ddagger}$ \\ University of East Anglia \\ Norwich, United Kingdom
}

Received 19.06.2020, received in revised form 07.09.2020, accepted 20.11.2020

\begin{abstract}
The response of a poroelastic ice cover to an external load is considered. The ice cover is modeled by a thin poroelastic floating plate within the linear theory of hydroelasticity. The porosity parameter is defined as the coefficient of proportionality of the velocity of liquid penetration into the plate and hydrodynamic pressure. The fluid under the plate is inviscid and incompressible. The flow caused by the ice deflection is potential. The external load is modeled by a localized smooth pressure. The two-dimensional problem of waves caused by a periodic external pressure on a floating porous-elastic plate is considered. The profiles of the generated waves are calculated for a given oscillation frequency of the amplitude of the external pressure. It was found that taking porosity into account leads to damping of oscillations in a distance from the external load.
\end{abstract}

Keywords: poroelastic ice plate, ideal fluid, external load, potential flow.

Citation: K.N. Zavyalova, K.A. Shishmarev, A.A. Korobkin, The Response of a Poroelastic Ice Plate to an External Pressure, J. Sib. Fed. Univ. Math. Phys., 2021, 14(1), 87-97.

DOI: $10.17516 / 1997-1397-2021-14-1-87-97$.

\section{Introduction}

The interaction of gravitational waves with thin porous structures is of considerable interest $[1,2]$. Such structures used as models for a wide range of physical problems, which include wave interactions with floating flexible breakwaters, large floating structures and thin ice sheets. At the moment, a lot of research has been done on the oscillations of floating structures and thin plates immersed in liquid [3-5]. In the study of dynamics of an ice cover, the most studied problem is determining of deflections of an elastic ice plate [6-9]. The problems were solved within the linear theory of hydroelasticity. Porosity of ice was not taken into account in these studies. A poroelastic ice plate model based on the Biot [10] theory of a poroelastic material is investigated in [11]. Dispersion relations of flexural gravity waves were investigated in [12] for one particular model of a poroelastic ice cover.

\footnotetext{
*kristina-zavyalova-1996@mail.ru

†shishmarev.k@mail.ru

$\ddagger$ A.Korobkin@uea.ac.uk

(c) Siberian Federal University. All rights reserved
} 
The focus of this article is on nonstationary oscillations of a poroelastic ice cover caused by an applied periodic pressure. The poroelastic ice cover is modeled by an elastic ice sheet with nonzero constant parameter of the porosity. The formulation of the problem is given in Section 1. The porosity of the ice is modeled through the kinematic condition at the ice-liquid interface using a term proportional to the fluid pressure [3]. Method of the solution is presented in Section 2. Numerical results are discussed in Section 3. The focus of the study is on the effect of the porosity on the deflections of the poroelastic ice plate. The main results of the work are presented in the conclusion and plan of the further work is discussed.

\section{Formulation of the problem}

Oscillations of a poroelastic ice plate caused by external pressure are considered. The fluid under the plate is inviscid and incompressible and is of finite depth $H(-H<y<0)$. The plate is infinitely long in the $x$ direction, where $(x, y)$ are Cartesian coordinates. The flow caused by the plate deflection is potential. The liquid can penetrate the plate on the liquid/plate interface: the liquid velocity along the normal to the plate $\left(V_{n L}\right)$ is equal to the normal component of the plate velocity $\left(V_{n s}\right)$ plus the velocity of the liquid penetrating into the plate along the normal to it $\left(v_{n}\right)$. The liquid velocity $\left(v_{n}\right)$ is considered proportional to the liquid pressure on the lower surface of the plate. If plate deflections $y=w(x, t)$ are small, then the kinematic condition has the form

$$
\frac{\partial \varphi}{\partial y}=\frac{\partial w}{\partial t}+v_{n}, \quad v_{n}=\alpha p(x, 0, t)
$$

where $p(x, 0, t)$ is the hydrodynamic pressure, $p=-\rho \varphi_{t}-\rho g w(x, t), w(x, t)$ is the vertical displacement (deflection) of the plate from the rest position, $\varphi(x, y, t)$ is the potential of the fluid flow velocity, $\alpha$ describes the porosity of the plate, $\rho$ is the fluid density, $g$ is the acceleration of gravity and the hydrodynamic pressure above the plate is assumed to be zero. In a more general case, $v_{n}=\alpha\left(p_{-}-p_{+}\right)$, where $p_{-}$is the hydrodynamic pressure on the lower surface of the plate, $p_{+}$is the hydrodynamic pressure above the fluid that has penetrated into the plate (arises in the case of the plate immersed in the fluid), $v_{n}>0$ if the liquid enters the plate from below. The assumption that the normal velocity of the penetrating fluid is proportional to the hydrodynamic pressure was used in many works, for example, in $[3,13,14]$. A similar model obtained using Darcy's Law is used in [5].

A periodic external load modeled by a pressure with an amplitude oscillating in time is considered. External pressure is applied to the upper surface of the plate and its position does not change over time. The problem is solved within the linear theory of hydroelasticity [6]. In this case the governing equations describing the problem are

$$
\begin{gathered}
M w_{t t}+E J w_{x x x x}=p(x, 0, t)-P_{\text {ext }}(x) \cos (\omega t), \\
\varphi_{x x}+\varphi_{y y}=0 \quad(-H<y<0), \\
\varphi_{y}=0 \quad(y=-H), \quad \varphi_{y}=w_{t}+\alpha p \quad(y=0), \\
p=-\rho \varphi_{t}-\rho g w \quad(y=0),
\end{gathered}
$$

where $M=\rho_{i} h_{i}$ is the mass of the ice per unit area, $\rho_{i}$ is the ice density, $h_{i}$ is the plate thickness, $P_{e x t}(x)$ is the function describing the shape of the external load, $E J=E h_{i}^{3} /\left(12\left(1-\nu^{2}\right)\right)$ is the flexural rigidity, $E$ is the Young's modulus, $\nu$ is the Poisson's ratio, $w_{x x x x}=\partial^{4} w / \partial x^{4}, \omega$ is the frequency of the load's amplitude oscillations. 
In general the equations (1)-(4) should be supplemented by the initial conditions and the condition for $x \rightarrow \infty$, as it was done, for example, in [8] for the problem of the large time response of an ice cover to a load moving along a channel. We shall study a stationary problem: the system (1)-(4) using the representation for unknown functions introduced below is reduced to a system of stationary equations, which is closed by the boundary condition for $x \rightarrow \infty$. As a consequence, the problem does not need initial conditions and their definition is not discussed in this article. The external load has a given form and periodic amplitude with a given frequency $\omega$. It is convenient to seek the functions $w(x, t), \varphi(x, y, t)$ and $p(x, y, t)$ in the form

$$
\begin{gathered}
w(x, t)=\operatorname{Re}\left[W(x) e^{i \omega t}\right], \\
\varphi(x, y, t)=\operatorname{Re}\left[i \omega \Phi(x, y) e^{i \omega t}\right], \\
p(x, 0, t)=\operatorname{Re}\left[P(x) e^{i \omega t}\right] .
\end{gathered}
$$

Seeking the solution in the form (5)-(7) is a common method in problems of oscillations of a thin plate with a load with a given constant frequency of the oscillating amplitude (see, for example, the method of the solution in [15]). Substitution of the functions (5)-(7) to the system (1)-(4) provides

$$
\begin{gathered}
-\omega^{2} M W+E J W_{x x x x}=P(x)-P_{e x t}(x), \\
P(x)=\rho \omega^{2} \Phi(x, 0)-\rho g W(x), \\
\Phi_{y}(x, 0)=W(x)+\frac{\alpha}{i \omega} P(x), \\
\nabla_{2}^{2} \Phi=0 \quad(-H<y<0), \quad \Phi_{y}(x,-H)=0 .
\end{gathered}
$$

The system of equations (8)-(11) is supplemented by the damping conditions of the ice deflections and fluid disturbances far away from the load

$$
W \rightarrow 0, \quad \Phi \rightarrow 0 \quad(x \rightarrow \infty)
$$

The solution to the problem (8)-(12) depends on the shape of the external pressure $P_{\text {ext }}(x)$, frequency $\omega$, fluid parameters $\rho, H$, parameters of the plate $M, E J$ and parameter of the porosity $\alpha$. We shall find the function $W(x)$ and use it to determine the deflections $w(x, t)$ of the poroelastic ice plate for some given parameters of the problem. The study in this article is focused on the effect of the parameter of the porosity $\alpha$ on the behavior of the poroelastic ice plate under the applied external periodic pressure. The correctness of the described problem is not considered in this article. In [16] the uniqueness of the classical solution of the problem of oscillations of a thin elastic plate in a channel for an arbitrary external load is proved and a review of the results on the correctness of the initial-boundary value problems of the joint dynamics of fluid and elastic ice cover is given. It is noted that the correctness of problems of the dynamics of a thin plate or fluid is well studied separately by many authors, when the problems of joint dynamics get much less attention. More results were obtained in the study of fluid filtration in porous ice cover based on Biot's theory. See, for example, articles [17,18]. In this article the process of fluid filtration in the ice plate is not taken into account. 


\section{Method of the solution}

The problem (8)-(11) is solved using the Fourier transform along the plate

$$
W^{F}(\xi)=\frac{1}{\sqrt{2 \pi}} \int_{-\infty}^{\infty} W(x) e^{-i \xi x} d x, \quad W(x)=\frac{1}{\sqrt{2 \pi}} \int_{-\infty}^{\infty} W^{F}(\xi) e^{i \xi x} d \xi
$$

The Fourier transform applied to the system of equations (8)-(11) provides the equation for the plate

$$
\left(\rho g-\omega^{2} M\right) W^{F}(\xi)+E J \xi^{4} W^{F}(\xi)=\rho \omega^{2} \Phi^{F}(\xi, 0)-P_{e x t}^{F}(\xi) .
$$

The Fourier image of the fluid velocity potential $\Phi^{F}(\xi, y)$ satisfies boundary problem

$$
\begin{gathered}
\Phi_{y y}^{F}-\xi^{2} \Phi^{F}=0 \quad(-H<y<0), \\
\Phi_{y}^{F}(\xi, 0)=W^{F}+\frac{\alpha}{i \omega}\left(\rho \omega^{2} \Phi^{F}-\rho g W^{F}\right), \\
\Phi_{y}^{F}(\xi,-H)=0 .
\end{gathered}
$$

The solution for $\Phi^{F}(\xi, y)$ is

$$
\Phi^{F}(\xi, y)=\Phi^{F}(\xi, 0) \cosh [\xi(y+H)] / \cosh (\xi H), \quad \Phi_{y}^{F}(\xi, 0)=\xi \tanh (\xi H) \Phi^{F}(\xi, 0) .
$$

Substitution of $\Phi_{y}^{F}(\xi, 0)$ to the transformed kinematic condition (14) gives

$$
\left[\xi \tanh (\xi H)-\frac{\alpha}{i} \rho \omega\right] \Phi^{F}(\xi, 0)=\left(1-\frac{\alpha}{i \omega} \rho g\right) W^{F}(\xi),
$$

and $\Phi^{F}(\xi, 0)$ can be written in the form

$$
\Phi^{F}(\xi, 0)=\frac{1+[\alpha \rho g / \omega] i}{\xi \tanh (\xi H)+\alpha \rho \omega i} W^{F}(\xi)
$$

Substituting $\Phi^{F}(\xi, 0)$ to the equation (13) we obtain the equation for determining $W^{F}(\xi)$

$$
\left[E J \xi^{4}+\rho g-\omega^{2} M-\rho \omega^{2} \frac{1+[\alpha \rho g / \omega] i}{\xi \tanh (\xi H)+\alpha \rho \omega i}\right] W^{F}(\xi)=-P_{e x t}^{F}(\xi), \quad W^{F}(\xi)=-\frac{P_{e x t}^{F}(\xi)}{Q(\xi)},
$$

where

$$
Q(\xi)=E J \xi^{4}+\rho g-\omega^{2} M-\rho \omega^{2} \frac{\xi \tanh (\xi H)+\alpha^{2} \rho^{2} g-\alpha \rho \omega i+\xi \tanh (\xi H)(\alpha \rho g / \omega) i}{\xi^{2} \tanh ^{2}(\xi H)+(\alpha \rho \omega)^{2}} .
$$

The function $Q(\xi)$ is symmetric, $Q(-\xi)=Q(\xi)$. Note that the functions $Q(\xi), W^{F}(\xi)$ and $P_{\text {ext }}^{F}$ are dimensional. For convenience and analysis of the convergence of the numerical solution we introduce the following parameters and dimensionless variables: $\tilde{\xi}=\xi H, \tilde{\alpha}=\alpha \rho \omega H$ is the dynamic porosity, $\Omega=\omega^{2} H / g, L_{c}=(E J /(\rho g))^{1 / 4}$ is the characteristic length [6]. In dimensionless variables $Q(\xi)$ reads

$$
\begin{gathered}
Q(\xi)=E J \frac{1}{H^{4}} \tilde{\xi}^{4}+\rho g-\omega^{2} M-\rho \omega^{2} H \frac{\tilde{\xi} \tanh (\tilde{\xi})+\alpha^{2} \rho^{2} g H-\alpha \rho \omega H i+\tilde{\xi} \tanh \tilde{\xi}(\alpha \rho g / \omega) i}{\left[\tilde{\xi}^{2} \tanh ^{2} \tilde{\xi}+(\alpha \rho \omega H)^{2}\right]} . \\
\frac{Q}{\rho g}=\frac{E J / \rho g}{H^{4}} \tilde{\xi}^{4}+1-\Omega \frac{M}{\rho H}-\frac{\Omega\left(\tilde{\xi} \tanh (\tilde{\xi})+\tilde{\alpha}^{2} / \Omega+i[\tilde{\xi} \tanh (\tilde{\xi}) \tilde{\alpha} / \Omega-\tilde{\alpha}]\right)}{\tilde{\xi}^{2} \tanh ^{2}(\tilde{\xi})+\tilde{\alpha}^{2}}=\tilde{Q}_{R}(\tilde{\xi})+i \tilde{Q}_{I}(\tilde{\xi}), \\
-90-
\end{gathered}
$$


where

$$
\tilde{Q}_{R}(\tilde{\xi})=\left(\frac{L c}{H}\right)^{4} \tilde{\xi}^{4}+1-\Omega \frac{M}{\rho H}-\frac{\tilde{\alpha}^{2}+\Omega \tilde{\xi} \tanh (\tilde{\xi})}{\left(\tilde{\xi} \tanh ^{2}(\tilde{\xi})+\tilde{\alpha}^{2}\right)}, \quad \tilde{Q}_{I}=\frac{\tilde{\alpha} \Omega-\tilde{\alpha} \tilde{\xi} \tanh (\tilde{\xi})}{\left(\tilde{\xi} \tanh ^{2}(\tilde{\xi})+\tilde{\alpha}^{2}\right)} .
$$

The function $W(x)$ is defined using the inverse Fourier transform applied to $W^{F}(\xi)$

$$
\begin{gathered}
W(x)=\frac{1}{\sqrt{2 \pi}} \int_{-\infty}^{\infty} \frac{-P_{e x t}^{F}(\xi)}{Q(\xi)} e^{i \xi x} d \xi=-\frac{1}{2 \pi} \int_{-\infty}^{\infty} P_{e x t}\left(x_{0}\right) \int_{-\infty}^{\infty} \frac{e^{-i \xi x_{0}+i \xi x}}{Q(\xi)} d \xi d x_{0}= \\
=-\frac{1}{2 \pi} \int_{-\infty}^{\infty} P_{e x t}\left(x_{0}\right) K\left(x-x_{0}\right) d x_{0}, \\
K(x)=2 \int_{0}^{\infty} \frac{\cos (\xi x)}{Q(\xi)} d \xi=\{\xi=\tilde{\xi} / H\}=2 \int_{0}^{\infty} \frac{\cos (\tilde{\xi} x / H) d \tilde{\xi}}{H \rho g\left[\tilde{Q}_{R}+i \tilde{Q}_{I}\right]}=\frac{2}{\rho g H}\left[K_{R}(x / H)-i K_{I}(x / H)\right],
\end{gathered}
$$

where $K(x)$ is a dimensional function, $K_{R}(x / H)$ and $K_{I}(x / H)$ are dimensionless functions calculated by the equations

$$
K_{R}(x / H)=\int_{0}^{\infty} \frac{\tilde{Q}_{R}(\tilde{\xi}) \cos (\tilde{\xi} x / H)}{\tilde{Q}_{R}^{2}+\tilde{Q}_{I}^{2}} d \tilde{\xi}, \quad K_{I}(x / H)=\int_{0}^{\infty} \frac{\tilde{Q}_{I}(\tilde{\xi}) \cos (\tilde{\xi} x / H)}{\tilde{Q}_{R}^{2}+\tilde{Q}_{I}^{2}} d \tilde{\xi} .
$$

Note that

$$
\tilde{Q}_{R}=O\left(\tilde{\xi}^{4}\right), \quad \tilde{Q}_{I}=O(1 / \tilde{\xi}), \quad \tilde{\xi} \rightarrow \infty,
$$

so we can expect that the integrals $K_{R}(x / H)$ and $K_{I}(x / H)$ will quickly converge in the numerical solution. The final equation for determining $W(x)$ is

$$
W(x)=-\frac{1}{\pi \rho g H} \int_{-\infty}^{\infty} P_{e x t}\left(x_{0}\right)\left[K_{R}\left(\frac{x-x_{0}}{H}\right)-i K_{I}\left(\frac{x-x_{0}}{H}\right)\right] d x_{0}=W_{R}(x)+i W_{I}(x) .
$$

The equation (15) is written in dimensional variables. Rewriting the equation for $W(x)$ in trigonometric form we get

$$
W(x)=|W(x)| e^{i \Theta(x)} .
$$

Accordingly, the deflections of the poroelastic ice plate under the action of periodic pressure are determined from the equation

$$
w(x, t)=\operatorname{Re}\left[W(x) e^{i \omega t}\right]=|W(x)| \cos [\Theta(x)+\omega t],
$$

where

$$
\operatorname{Re}\left[W(x) e^{i \omega t}\right]=\operatorname{Re}\left[\left(W_{R}(x)+i W_{I}(x)\right) e^{i \omega t}\right]=W_{R}(x) \cos (\omega t)-W_{I}(x) \sin (\omega t)
$$

and the function $\Theta(x)$ is defined by

$\cos (\Theta(x))=W_{R}(x) /|W(x)|, \quad \sin (\Theta(x))=W_{I}(x) /|W(x)|, \quad|W(x)|=\sqrt{\left(W_{R}(x)\right)^{2}+\left(W_{I}(x)\right)^{2}}$.

It is expected that the parameter $\Theta(x)$ will be related to the wave number of waves propagating from the load in the poroelastic plate. 


\section{Numerical results}

Calculations of the deflections of the poroelastic ice plate under the action of the external pressure were made for following parameters of the problem: $H=2 \mathrm{~m}, E=4.2 \cdot 10^{9} \mathrm{~Pa}, \nu=0.3$, $h_{i}=0.1 \mathrm{~m}, \rho=1024 \mathrm{~kg} / \mathrm{m}^{3}, \rho_{i}=917 \mathrm{~kg} / \mathrm{m}^{3}, g=9.8 \mathrm{~m} / \mathrm{s}^{2}, \omega=1 \mathrm{~s}^{-1}$. The shape of the external load $P_{\text {ext }}(x)$ in our calculations is given in the form

$$
\begin{gathered}
P(x)=P_{0} P_{1}(x), \\
P_{1}(x)=(\cos (\pi c x)+1) / 2(c|x|<1), \quad P_{1}(x)=0 \quad(c|x| \geqslant 1),
\end{gathered}
$$

where $c=1, P_{0}=1000 \mathrm{~Pa}$.

The function $W(x)$ is calculated using the equation (16). In calculating the integrals for the poroelastic plate we need to investigate the functions under the integrals and choose the integration step carefully. The integrands in (16) have no singularities due to the term with the porosity, but as the parameter $\alpha$ decreases these functions tend to singular functions with a singularity at some point in $\xi$. For example, let us consider the function $\tilde{Q}_{R} /\left(\tilde{Q}_{R}^{2}+\tilde{Q}_{I}^{2}\right)$, which appears in the integral $W_{R}(x)$. This function is shown in Fig. $1 \mathrm{a}$ for $\alpha=0.1$, in Fig. $1 \mathrm{~b}$ for $\alpha=0.01$ and in Fig. $1 \mathrm{c}$ for $\alpha=0.0001$. The distance between the negative and positive peaks of this function decreases for large $(>0.1)$ and small $(<0.0001)$ values of the parameter $\alpha$. In all presented cases this function quickly damped. To accurately calculate $W(x)$ the function $\tilde{Q}_{R} /\left(\tilde{Q}_{R}^{2}+\tilde{Q}_{I}^{2}\right)$ was calculated for cases (a) and (c) with $2 \cdot 10^{5}$ number of steps on the interval from 0 to 1 in $\tilde{\xi}$. In (b) case the number of steps can be reduced without the loss of accuracy.
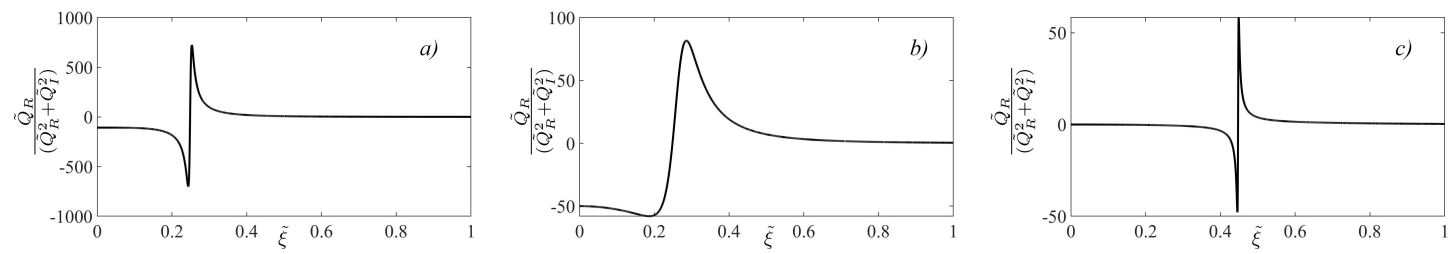

Fig. 1. Function $\tilde{Q}_{R} /\left(\tilde{Q}_{R}^{2}+\tilde{Q}_{I}^{2}\right)$ for $\alpha=0.1$ (a), for $\alpha=0.01$ (b) and for $\alpha=0.0001$ (c)

In all following presented results we use the following parameters of the integration: the integrals over $\tilde{\xi}$ on the interval from 0 to 1 are calculated with $2 \cdot 10^{5}$ number of steps, then from 1 to 10 with $10^{4}$ number of steps. All integrals were calculated using the trapezoidal rule with appropriate steps on the intervals from 0 to 1 and from 1 to 10 . In Section 2 it is noted that the calculated integrals will decay quickly due to the properties of the functions $\tilde{Q}_{R}$ and $\tilde{Q}_{I}$ when $\tilde{\xi}$ tends to infinity. A series of calculations with different upper limits $(10,20,30)$ showed that for the considered values of the parameter of the porosity increasing the value of the upper limit more than 10 does not lead to noticeable changes in the ice deflections $W(x)$. This is confirmed by the estimates of these integrals. For example, in the integral $K_{R}$ for $\tilde{\xi}>1$ one can use the estimate $\frac{\tilde{Q}_{R}(\tilde{\xi}) \cos (\tilde{\xi} x / H)}{\tilde{Q}_{R}^{2}+\tilde{Q}_{I}^{2}}<1 / \tilde{\xi}^{4}$. The ice deflections $w(x, t)$ calculated by the equation $(16)$ for $t=0 \mathrm{~s}$ are shown in Fig. 2. The solid line shows the case with $\alpha=10^{-8}$, the dotted line shows the case with $\alpha=10^{-6}$. The horizontal lines show the amplitude level of the generated waves. Note that in the presence of porosity, waves in the ice sheet will propagate from the load due to the presence of an imaginary part in determining $W(x)$. For the interval in $x$ under consideration, in accordance with Fig. 2, we can assume that for finite $x$ the solution converges 
as the value of $\alpha$ decreases. Ice deflections for $\alpha=10^{-8}$ can be interpreted as an approximation of the ice deflections of an elastic plate with zero porosity for similar parameters of the problem. For a complete analysis, it is necessary to solve the problem (8)-(12) with $\alpha=0$ separately.

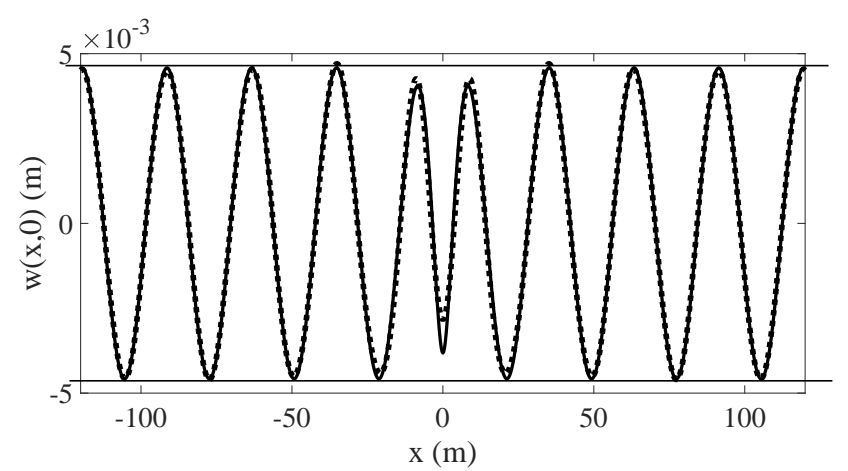

Fig. 2. The deflections of the poroelastic ice plate for $\alpha=10^{-8}$ (solid line) and for $\alpha=10^{-6}$ (dotted line), $t=0 \mathrm{~s}$

To study the effect of porosity on the deflections of the poroelastic ice plate some calculations were carried out with different numbers of $\alpha$. The choice of the value of $\alpha$ is not obvious. In [5] the porosity parameter is denoted as $P, P=\omega K \rho /\left(\nu h_{i}\right)$, where $K$ is the permeability of the ice and $\nu$ is the dynamic viscosity of the fluid. Matching the parameter $P$ with the parameter $\alpha$ considered in this article by converting the kinematic condition to one form (equation $2 \mathrm{a}$ in [5]), we obtain the relation $P=\alpha \omega \rho$. In the work [5], the calculations were made for the values $P H=0,0.5$ and 1 . For the model considered in this article, these values correspond to $\alpha=0, \alpha \approx 0.00025$ and 0.0005 , respectively. The results of the calculations of the deflections are shown in Fig. 3 for $\alpha=10^{-8}, 0.00005,0.0001$ and 0.00025 . It was found that the increase in the parameter of the porosity increases the amplitude of the deflections in the load area, but decreases the amplitude at a distance from the load. With the increase in the parameter of the porosity the width of disturbed area of the ice cover decreases as well as the length and rate of decaying of the hydroelastic waves propagating from the load. The calculated ice deflections $w(x, t)$ for any nonzero value of the parameter of the porosity tend to 0 when $x \rightarrow \infty$, which means that the condition (12) with the nonzero parameter of the porosity is natural.

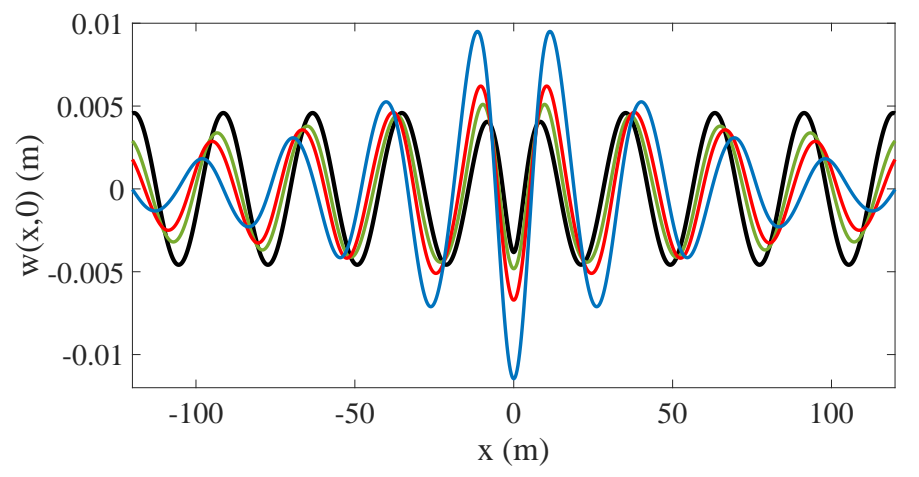

Fig. 3. The deflections of the poroelastic ice plate for $t=0 \mathrm{~s}$. Black line shows the case with $\alpha=10^{-8}$, green line $-\alpha=0.00005$, red line $-\alpha=0.0001$ and blue line $-\alpha=0.00025$ 

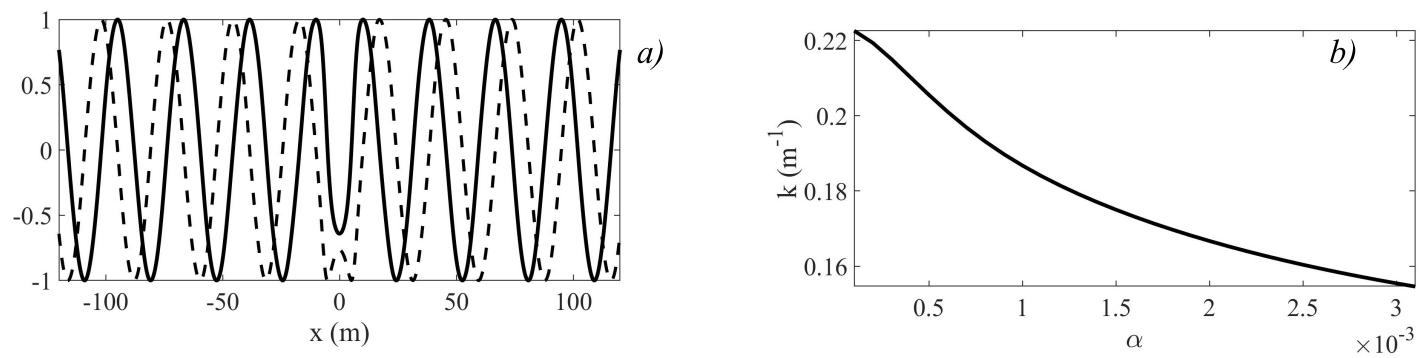

Fig. 4. Functions $\cos (\Theta(x))$ and $\sin (\Theta(x))$ for $\alpha=0.0001$ (a). The wave number $k$ as a function of $\alpha(\mathrm{b})$

As shown in Fig. 3, the deflections of the poroelastic ice plate have a wave structure. Moreover, the length of these waves differs for different values of the parameter of the porosity. To determine the length of the generated waves on the considered fixed segment in $x$, the following algorithm is proposed. The wavelength depends on the function $\Theta(x)$. This function is the solution of the equations $\cos (\Theta(x))=|W(x)| / W_{R}(x), \sin (\Theta(x))=|W(x)| / W_{I}(x)$. The functions $\cos (\Theta(x))$ and $\sin (\Theta(x))$ are shown in Fig. 4 for $\alpha=0.0001$. The function $\Theta(x)$ is calculated as a periodic function with values from 0 to $2 \pi$ using the mentioned equations. This function is even, $\Theta(x)=\Theta(-x)$. Consider $\Theta(x)$ for positive values of $x, x>0$. We introduce the function $\tilde{\Theta}(x)=\Theta(x)+2 \pi(m-1)$, where $m$ corresponds to the $m$-th periodic part of the function $\Theta(x)$ in positive $x$ direction, starting from $x=0$. The function $\tilde{\Theta}(x)$ satisfies the equations $\cos (\tilde{\Theta}(x))=|W(x)| / W_{R}(x), \sin (\tilde{\Theta}(x))=|W(x)| / W_{R}(x)$ and can be approximated by the linear function $k x+b$, where the parameter $k$ is the wavenumber of the considered wave. These wave numbers $k$ are shown in Fig. $3 \mathrm{~b}$ as a function of the parameter of the porosity $\alpha$. For the presented wave numbers we used the approximation of the function $\Theta(x)$ on the interval $[0,120]$. The approximated function $\tilde{\Theta}(x)$ for positive $x$ for different values of $\alpha$ are shown in Fig. 5 .

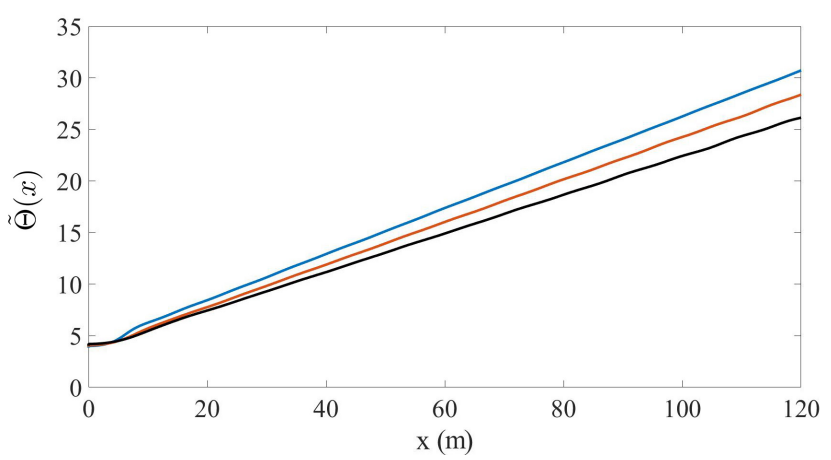

Fig. 5. Approximated function $\tilde{\Theta}(x)$ for positive $x$ with $\alpha=0.001$ (black line), $\alpha=0.0005$ (red line) and $\alpha=0.0001$ (blue line)

The deflections of the poroelastic ice plate $w(x, t)$ at $t \in[0,6]$ are shown in Fig. 6 in the form of the surfaces in $(x, t)$ domain with different values of $\omega$ for $\alpha=0.0001$. The case for $\omega=1 \mathrm{~s}^{-1}$ is shown in Fig. 6 a, the case for $\omega=2 \mathrm{~s}^{-1}$ is shown in Fig. 6 b. The period of the oscillations of $w(x, t)$ in $t$ for $\omega=1 \mathrm{~s}^{-1}$ is $2 \pi$ (Fig. $6 \mathrm{a}$ ), and for $\omega=2 \mathrm{~s}^{-1}$ is equal to $\pi$ (Fig. $6 \mathrm{~b}$ ). The increase in the frequency decreases the disturbed area of the plate, amplitude of the ice deflections and wavelength of the hydroelastic wave in the plate. 

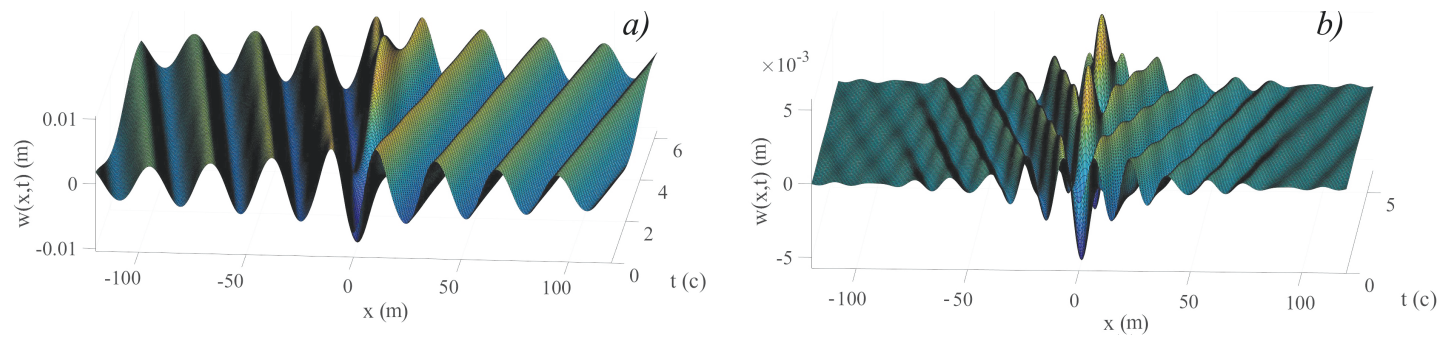

Fig. 6. The deflections of the poroelastic ice plate $w(x, t)$ in the form of the surfaces in $(x, t)$ domain for $\omega=1 \mathrm{~s}^{-1}$ (a) and for $\omega=2 \mathrm{~s}^{-1}$ (b), $t \in[0,6]$

\section{Conclusion}

The non-stationary oscillations of the poroelastic ice cover caused by the external periodic pressure are considered. The ice cover is modeled by a thin poroelastic floating plate with a given parameter of the porosity. The parameter of the ice porosity is defined as the coefficient of proportionality between the velocity of the liquid penetrating into the plate and the hydrodynamic pressure. The formulation of the problem statement and method of the solution are presented. The problem is solved using the Fourier transform along the plate. The numerical convergence of the obtained integrals is investigated.

The effect of the porosity on the deflections of a poroelastic ice plate is investigated. Porosity leads to damping of oscillations at a distance from the applied pressure. It is shown that the ice deflections have a wave structure. It was found that the increase in the porosity parameter increases the deflections of the poroelastic plate near the applied pressure, decreases the ice deflections at a distance from it, decreases the width of the disturbed area of the plate. We associate this behavior of the ice cover with the dissipation caused by the porosity. Porosity increases deflections under the load because of the applied pressure, and reduces deflections away from the load, where dissipation due to the porosity is important. The porosity also increases the length of the hydroelastic wave propagating from the applied pressure. The method of calculating the length of such a wave is proposed, and calculations are performed for different values of the porosity parameter. To verify this fact, it is necessary to investigate the dispersion relations of the generated waves, in particular, with zero porosity. It is shown that the increase in the frequency of oscillations of the applied pressure's amplitude decreases the ice deflections, the disturbed area of the plate, and the wavelength. We associate this with the fact that the dissipation is usually higher for high frequencies. High frequency oscillations decay faster and the wavelength should decrease with the frequency.

The obtained results make it possible to evaluate the effect of the porosity on the behavior of the ice cover under the action of the applied external loads. In this work the effect of a periodic load is investigated, however, the load can move and have a different shape, then in this case the problem requires additional research. Most of the articles that consider models of the porous plates similar to one used in this article are devoted to the study of wave scattering and energy dissipation $[5,19]$. The external load is not taken into account in these works. In the work [19] wave scattering by a poroelastic plate of any shape in a three-dimensional formulation was investigated and it was found that the increase in the porosity parameter decreases the amplitudes of the deflections of the poroelastic plate, which is in good agreement with our results for the ice deflections away from the load. For further comparison of the obtained results, it is necessary to investigate the considered problem in a three-dimensional formulation taking 
into account the ice plate of finite length and different boundary conditions. Also, for a better understanding of the effect of porosity, it is necessary to investigate the considered problem with the porosity coefficient equal to zero and compare the results. In this case, the damping conditions is not guaranteed and the Fourier transform cannot be used. To find a solution in this case, the vertical mode method can be used [20].

This work was carried out according to project MK-204.2020.1 "Initial-boundary value problems for equations of fluid motion in poroelastic media and their application in the dynamics of snow-ice cover" with the support of a grant from the President of the Russian Federation.

\section{References}

[1] S.Zhenga, M.Meylan, G.Zhua, D.Greavesa, G.Iglesiasc, Wave scattering from multiple circular floating porous elastic plates, The 35th International Workshop on Water Waves and Floating Bodies, 26-29 April, 2020, Seoul, Korea, 2020.

[2] D.Mondal, S.Banerjea, Scattering of water waves by an inclined porous plate submerged in ocean with ice cover, Quarterly Journal of Mechanics and Applied Mathematics, 69(2016), no. $2,195-213$.

[3] A.T.Chwang, A porous-wavemaker theory, Journal of Fluid Mechanics, 132(1983), 395-406.

[4] D.V.Evans, M.A.Peter, Asymptotic reflection of linear water waves by submerged horizontal porous plates. Journal of Engineering Mathematics, 69(2011), 135-154.

[5] M.H.Meylan, L.G.Bennetts, M.A.Peter, Water-wave scattering and energy dissipation by a floating porous elastic plate in three dimensions, Wave Motion, 70(2017), 240-250.

[6] V.Squire, R.Hosking, A.Kerr, P.Langhorne, Moving loads on ice, Kluwer Academic Publishers, 1996.

[7] K.A.Shishmarev, T.I.Khabakhpasheva, A.A.Korobkin, The response of ice cover to a load moving along a frozen channel, Applied Ocean Research, 59(2016), 313-326.

[8] T.I.Khabakhpasheva, K.A.Shishmarev, A.A.Korobkin, Large-time response of ice cover to a load moving along a frozen channel, Applied Ocean Research, 86(2019), 154-165.

[9] V.A.Squire, Moving loads on ice, Ocean wave interactions with sea ice: a reappraisal Annual Review of Fluid Mechanics, 52(2020), 37-60. DOI: 10.1146/annurev-fluid-010719-060301

[10] M. A. Biot, Theory of Finite Deformations of Porous Solids, Indiana Univ. Math, 21(1972), 597-620.

[11] L.A.Taber, A.M.Puleo, Poroelastic Plate and Shell Theories, Mechanics of Poroelastic Media. Solid Mechanics and Its Applications, 35(1996), 323-337.

[12] H.Chen, R.P.Gilbert, P.Guyenne, Dispersion and attenuation in a porous viscoelastic model for gravity waves on an ice-covered ocean, European Journal of Mechanics-B/Fluids, 78(2019), 88-105.

[13] G. Taylor, Fluid Flow in regions bounded by porous surfaces, Proceedings of the Royal Society A, 234(1956), 456-475. 
[14] A.T.Chwang, A.T.Chan, Interaction between porous media and wave motion, Annu. Rev. Fluid Mech, 30(1998), no. 1, 53-84.

[15] L.A.Tkacheva, Action of a local time-periodic load on an ice sheet with a crack, Journal of Applied Mechanics and Technical Physics, 58(2017), no. 6, 1069-1082.

DOI: $10.1134 /$ S002189441706013X

[16] K.A.Shishmarev, A.A.Papin, Uniqueness of a solution of an ice plate oscillation problem in a channel, Journal of Siberian Federal University. Mathematics and Physics, 11(2018), no. 4, 449-458. DOI: 10.17516/1997-1397-2018-11-4-449-458

[17] M.A.Tkacheva, Solvability of initial boundary value problem for the equations of filtration in poroelastic media, Journal of Physics: Conference Series, 722(2016), 012037.

DOI: $10.1088 / 1742-6596 / 722 / 1 / 012037$.

[18] A.A.Papin, M.A.Tokareva, Problems of heat and mass transfer in the snow-ice cover, IOP Conference Series: Earth and Environmental Science, 193(2018), no. 1, 012055.

DOI: $10.1088 / 1755-1315 / 193 / 1 / 012055$

[19] S.Zheng, M.Meylan, L.Fan, D.Greaves, G.Iglesias, Wave scattering by a floating porous elastic plate of arbitrary shape: A semi-analytical study, Journal of Fluids and Structures, 92(2020), 102827. DOI: 10.1016/j.jfluidstructs.2019.102827

[20] A.A.Korobkin, S.Malenica, T.Khabakhpasheva, The vertical mode method in the problems of flexural-gravity waves diffracted by a vertical cylinder, Applied Ocean Research, 84(2019), $111-121$.

\title{
Поведение пороупругой ледовой пластины под действием приложенного давления
}

\author{
Кристина Н. Завьялова \\ Константин А. Шишмарев \\ Алтайский государственный университет \\ Барнаул, Российская Федерация \\ Александр А. Коробкин \\ Университет Восточной Англии \\ Норидж, Соединенное Королевство
}

\begin{abstract}
Аннотация. Рассматриваются колебания пороупругого ледового покрова под действием приложенной внешней нагрузки. Лед моделируется тонкой пороупругой плавающей пластиной в рамках линейной теории гидроупругости. Параметр пористости определяется как коэффициент пропорциональности скорости проникновения жидкости в пластину и гидродинамического давления. Жидкость под пластиной идеальная и несжимаемая. Течение, вызванное прогибом пластины, считается потенциальным. Внешняя нагрузка моделируется локализованным гладким пятном давления. Рассмотрена двумерная задача о волнах, вызванных периодическим внешним давлением на плавающую пороупругую пластину. Вычислены профили генерируемых волн при заданной частоте осцилляций амплитуды внешнего давления. Определено влияние пористости на прогибы пороупругого ледового покрова. Получено, что учет пористости приводит к затуханию колебаний в отдалении от области приложенного давления.
\end{abstract}

Ключевые слова: пороупругая ледовая пластина, идеальная жидкость, внешняя нагрузка, потенциальное течение. 